\title{
Our global-scale cognitive map: is it influenced by our place of residence or education?
}

\author{
Lieselot Lapon $^{\text {a, } * \text {, Kristien Ooms }}{ }^{\text {a }}$, Philippe De Maeyer ${ }^{\mathrm{a}}$ \\ ${ }^{a}$ Ghent University, Department of Geography, Lieselot Lapon, Lieselot.Lapon@UGent.be, Kristien Ooms, \\ Kristien.Ooms@UGent.be,Philippe De Maeyer,Philippe.DeMaeyer@UGent.be \\ * Corresponding author
}

Keywords: Cognitive map, Cartography, Education

\begin{abstract}
:
Cognitive maps are defined as internal representations of the environment or of the globe. Several scientists of various disciplines (e.g. psychology, geography) have conducted cognitive research on issues related to maps aiming to understand how humans create and utilize mental representations of the earth. Concluding that several elements, such as wayfinding tasks, verbal explanations or maps contribute to the creation of these representations. Moreover, map projections of world maps and their distortions are presumed as a crucial factor when creating our global-scale cognitive map. Nevertheless, previous research (of Battersby and Montello in 2006 at University of Santa Barbara and Lapon in 2017 at Ghent University) could not provide any proof for the existence of an influence of map projections on our globalscale cognitive map. To go deeper into this topic, we want to research any other possible influence on our cognitive map. For example, does the place where you were educated or the place where you live and work has an impact on the development of your mental map? Are these influences defined by the age, the gender or the educational level of people?

There are several measures to define the accuracy of our cognitive map: e.g. the distances between places or cities; the topologic relations between places, countries or other geographical elements; the land areas of countries and continents. The test developed for this research focussed on the latter one: participants are requested to estimate the size of one country or continent compared to another one (Figure 1). Therefore, an interactive playful online test was developed. The test is available in eight languages and consists of an instructive movie, ten pairs of countries or continents to estimate, a survey and a feedback tool which gives a score.
\end{abstract}

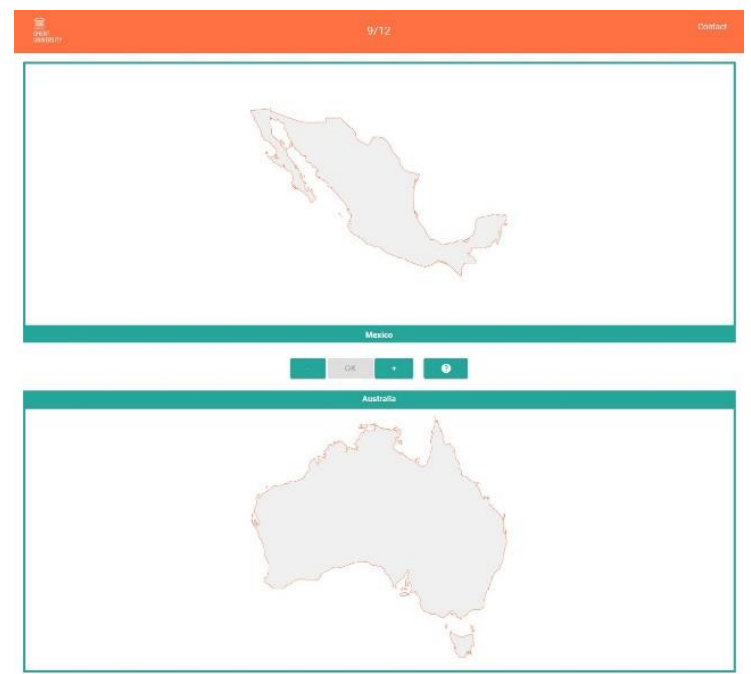

Figure 1. Setup of the online test: with the minus and plus participants can estimate the real size of both countries

This setup permits to collect data for our research, but it is also developed as a tool to create awareness about our misperception of region sizes among the participants. Furthermore, the test can easily be used in the classroom to introduce subjects such as map projections, cartography, world problems... By using personal contacts, mailing lists of the university, and social media, 100,000 fully completed tests were collected in two months' time, from more than 150 different countries worldwide. For every estimation the relative estimated accuracy is calculated, this value gives an impression to what extent people over- or underestimate one region compared to another. Its absolute value is an indication of how good people are in estimating the real size of countries or continents. These values were used to calculate differences between the participants, and between groups of participants. Besides their gender, age, profession, educational level and cartographical background, people were asked to define the country where they received their education and the country where they are living now. 
Nine countries, with more than 400 participants, spread over the globe, were selected for further analyses. The preliminary results show that there is a low variation between the averages for each country of education or place of living separately. So, it seems that the ability to estimate the size of countries or continents is not determined by the place where one lives or received one's education. In contrast, personal characteristics are more defining for the accuracy of the estimations. General results indicate that on the one side men achieve a better score than women, and on the other side young people between 12 and 18 years old perform quite better, in contrast with the participants older than 71 years. Also, the people with a higher educational level or a broader cartographical background perform better.

The place where you grow up, get educated or where you live, does not have a substantial influence on the accuracy of the estimations. Surprisingly, personal characteristics and qualities seem to play a more determining role, worldwide, in the development of geographical knowledge or more specific, in the evolvement of the cognitive map. To support these preliminary results, the analyses will be extended and the group of participants further delimited. Furthermore, a geographical dataset with more than 100,000 participants offers plenty of possibilities that will be exploited in the upcoming months. 